\title{
Critical issues of Emergency Medicine organization in Italy
}

\author{
Daniele Coen, Ivo Casagranda \\ Academy of Emergency Medicine and Care (AcEMC), Italy
}

\section{Introduction}

Over the last 50 years, Emergency Medicine (EM) has established itself as a medical specialty in many countries. The recognition of EM as a specialty in the USA dates back to 1972 and in Italy the establishment of the School of Emergency Medicine is now approaching 10 years. ${ }^{1,2}$

If the core curriculum of the different schools is similar in its main contents, the organization of the Emergency Departments (ED) is often different, sometimes very different, in several countries and within the same country, among large and small hospitals.

In Italy there is a plurality of regional models that is expressed in particular by the model of the Emergency Regional Agencies in three regions (Lombardy, Lazio and Sardinia), that keep separate the management of the Emergency Medical Services (EMS) from ED and by models that integrate the two systems (the most consolidated experiences are those of Emilia Romagna and Tuscany).

Even the qualification and the employment contracts of medical personnel involved in the management of emergencies, both in ED and in EMS, are very different.

If on the one hand there seems to be a widespread agreement on the fact that the holding of a specialty diploma in emergency medicine and a dependency contract should be the objective to aim for, on the other hand the shortage of specialized physicians and the presence of significant economic constraints make this perspective very distant.

\section{The scenario}

The scenarios that influence the organization of the EDs are constantly evolving under the pressure of external forces such as population aging, new immigration, choices of economic policy and the development of technology.,4

\footnotetext{
Correspondence: Daniele Coen, Academy of Emergency Medicine and Care (AcEMC), Via Salvatore Maugeri 10, 27100 Pavia, Italy.

Tel.: +39.0382.592794 - Fax: +39.0382.24506.

E-mail: daniele.coen@ospedaleniguarda.it

Key words: Emergency Medicine organization; Italy.

Received for publication: 13 September 2018.

Accepted for publication: 18 September 2018.

This work is licensed under a Creative Commons Attribution 4.0 License (by-nc 4.0).

(C) Copyright D. Coen and I. Casagranda, 2018

Licensee PAGEPress, Italy

Emergency Care Journal 2018; 14:7825

doi:10.4081/ecj.2018.7825
}

The decision to transfer the management of a growing share of acute patients from the hospital to the primary care, carried out with an otherwise aware and structured way in many Italian regions, is the most evident among the critical results of the new conditions in which the Italian Healthcare System (IHS) is forced to operate.

At the basis of this trend, which we believe is meant to increase rapidly in the years to come, there are in particular three phenomena of clinical/organizational feature.

The first is the growth in the request of hospital admission for patients with acute on chronic clinical conditions. These are mainly elderly patients, who would need regular and pro-active followup by their general practitioner, to recognize early signs of worsening in order to avoid hospitalization.

The second is the increasing use of the ED as a reference point for the management of minor urgencies, theoretically (but not easily) manageable elsewhere.

The third, is the growth in the number of patients that have not access to the IHS, in particular the new immigrants, many of whom are not insured because of the lack of a residence permit.

\section{Primary care response}

The response of the primary care organization has not yet given the expected results. The causes of this situation are numerous, ranging from the lack of practice by general practitioners in managing clinically complex patients, to the lack of a basic diagnostic availability (ultrasound, ECG, point of care testing) in their outpatient clinics. Moreover, the increasing cost and waiting list for many exams and consultancies induce a growing part of the population, especially in the areas with major social problems, to reduce the quantity and quality of clinical controls.

The transfer to the out-of-hospital areas of acute on chronic patients has taken different names and methods (chronicity manager, community hospitals, health houses, at home hospitalization, migrant clinics, etc.), but keeps at this moment a sporadic and marginal nature and lacks documentation of effectiveness/efficiency.

Although there are international examples of how the management of acute patients at home can find operating methods and have outcomes that satisfy patients and operators, most of them are far from our experience and organization.

This should not exempt us from reflecting on the inadequacy of our schemes, in particular the administrative rigidity, the existing constraints in the arrangement of healthcare personnel between in- hospital and out-of-hospital areas, the clinical role of nurses and the allotment of technological resources.

The specific issue of migration medicine continues to find no institutional responses other than the hospital, whose burden is only slightly mitigated by the meritorious work of non-profit voluntary associations. 


\section{The inadequacy of the classic emergency depart- ment model in a time of crisis}

Since the ' 80 s the relentless growth of medical knowledge has induced a parallel growth in the number of medical specialties and subspecialties that have influenced patient flow within the hospital. Things are now quickly changing because decreasing resources have caused an important reduction both of hospital beds and of specialists. As a consequence, one can see the return to general medical wards of patients that would have once been admitted to a subspecialty, and internists have to take care of patients suffering from a wider number of acute clinical conditions.

In this context, emergency physicians have been asked to control even more strictly the number of patients they admit, and to follow for a longer time acute patients waiting for an inpatient bed.

In many hospitals this has brought about the development of intermediate care units, where emergency physicians take care of patients not so unstable to deserve an ICU bed, and yet sufficiently acute to require strict monitoring and aggressive treatment. Today, in many hospitals, emergency physicians have acquired skills that up to a few years ago used to be of specialist pertinence only.

The efficient working of the EDs has allowed, through the ' 90 s and the first years of the new millennium, to significantly reduce the number of admissions, saving money and coming to terms with the fall in hospital beds. Today, the lack of beds and doctors is such that good will and hard work are not enough, and the system risks to enter a loop of worsening quality and unwanted negative outcomes.

Considering that, as we have discussed, times don't seem ripe for the transfer to primary care of patients with acute on chronic conditions, more thought should be given to the role of the ED and of the emergency physicians. In particular we should reconsider the model of an ED devoted only to the immediate evaluation and treatment of emergencies or minor ailments, pending admission for all patients in need of a stay longer than 4-6 hours. While a vast majority of minor problems could quite easily find their way to the renewed premises of primary care (or even to dedicated nurse run out-patient clinics), the ED should follow for a longer time, in well equipped units, patients of higher complexity and severity. While waits of 24-48 hours for a hospital bed are, for the time being, an intolerable tribute to inefficiency and a waste of time at high risk of complications, a similar period could be sufficient to study, stabilize and discharge home a high percentage of patients that today get usually admitted. Of course, this might become real only in the presence of adequate spaces, staff (both as to number and competence) and technology.

\section{New roles and beautiful exit of the experienced emergency physician}

Amongst the most worrisome aspects of the present condition of Italian emergency physicians are their increasing age and their very scanty chances of professional career. The perspective of having to work in shifts and over week ends up to the age of 65 in the chaotic and very demanding environment of the ED, is a problem both for doctors and the system. It is thus necessary to start reasoning about a possible beautiful exit for emergency physicians. In particular, career progression should be rendered possible for experienced and well trained 40-50 years old emergency physicians, with at least 10 years of work in the ED, observation units or intermediate care units, who have developed the best method- ological and technical skills of their role. It is likely that, as time goes by, these figures will also have gone through formal university education in emergency medicine.

The challenge comes from the growing number of physicians whose competences need to be addressed to the best before further ageing, weariness and deluded expectations drain their opportunities or even turn them into difficult to handle problems. A fulltrained emergency physician is a resource for the system that could help the development of many different areas of acute health care. For instance such a figure could: i) Take on a leading role within his/her own ED as a department or area chief, devoting part of his/her time to clinical consulting and part to the education and training of younger colleagues; ii) Move to an internal medicine ward, contributing to renewing the lost competences in dealing with acute patients and with a wider number of medical conditions; iii) Move from a teaching hospital to more peripheral institutions, to take responsibility of the ED and help spread the most advanced concepts and organization of modern emergency medicine; iv) $\mathrm{Be}$ assigned a post in general practice, and help in the development of group practices, health houses, at home hospitalization and community hospitals. This would probably need to modify the present criteria for the assignment of general practices, but it should not be a problem in a country like Italy where tens of thousands of vacant practices are foreseen in the years to come.

\section{A feasible open future}

A second strictly linked area for innovation is physicians' turn over in Emergency Departments. At variance with other countries, in Italy young doctors who get a contract in the ED find it difficult to move later towards other departments. At the same time, rotations in emergency for residents in internal medicine and other medical and surgical grad-schools are usually short and scarcely attended.

A new, less static model, should be imagined and developed also in the perspective of the establishment of teaching hospitals. The key element we should work about is the presence of an adequate number of young doctors in training, under the constant supervision of one or more fully trained emergency physicians. Trainees in their last year of internal medicine, geriatrics, cardiology, general surgery should spend at least six months as ED residents and they might if necessary be asked to work there for one more year after attaining their specialty degree. Residents in emergency medicine might stay longer, starting a process that could lead them to a consulting role within 3 to five years. In this way well trained physicians over 40 could benefit of more favorable shifts and act as consultants, thus seeing their competences recognized beyond possible income benefits. Applying this model, which is quite similar to that of English speaking countries, a progressive rejuvenation of the Italian EDs could be expected, while doctors who have developed advanced competences in dealing with acute conditions could be redistributed throughout the health system within and outside hospitals. Selection for a hospital position in emergency medicine should be differentiated between a basic and a consultant role, with growing economic compensations that would favor the move of well trained and expert physicians out of big city hospitals toward minor institutions. These small hospitals would otherwise be forced (as they are unluckily today) to hire doctors with inadequate training and an even lower vocation to serve as an emergency physician. 


\section{Conclusions}

The management of acute patients is an evolving issue in Italy as in other countries. There is a pressure to develop less expensive patient centered health systems, and for this reason possible alternatives to hospital care are being considered and experimented.

In this perspective the role of emergency medicine and its professionals may be pivotal. It is necessary to explore new organizational models that look beyond the fence of corporatism and rigid boundaries between specializations in order to favor an osmosis of competences and a better functioning of both in and out of hospital acute care services.

\section{References}

1. Suter RE. Emergency medicine in the United States: A systematic review. World J Emerg Med 2012;3:5-10.

2. Repetto C, Casagranda I, Overton D, Gai V. Emergency medicine: the Italian experience. Ann Emerg Med 1998;32:248-52.

3. Wiler JL, Harish, Zane RD. Do hospitals still make sense? The case for decentralization of health care. Available from: https://catalyst.nejm.org/hospitals-case-decentralizationhealth-care/

4. de Belvisa AG, Ferrè F, Specchia ML, et al. The financial crisis in Italy: Implications for the healthcare sector. Health Policy 2012;106:10-6. 